\title{
The Success of Surgical and Medical Approach in the Treatment of Cesarean Scar Pregnancy
}

\author{
Sara Masihi ${ }^{1}$, Mahin Najafian ${ }^{1}$, Mojgan Barati $^{1}$, Zohreh Mirfazli $^{1 *(D)}$
}

\begin{abstract}
Objectives: The ectopic pregnancy in the cesarean section (CS) scar (CSP) is one of the potentially dangerous and late complications of CS. The current study aimed to evaluate the therapeutic approaches in managing CS scar ectopic pregnancy.

Materials and Methods: This cross-sectional study was carried out on 63 CSP patients. The treatment approach was considered based on the shape and nature of the gestational sac (GS) during ultrasound evolutions. Patients' demographic data, clinical manifestations, and systemic drug treatment (methotrexate, MTX), as well as the type of surgery, hospitalization times, and treatment-related complications were evaluated, followed by comparing the treatment efficacy in different approaches based on the human chorionic gonadotropin (hCG) resolution time.

Results: The mean age of the subjects was 34.2 \pm 5.1 years (within the range of 22-44 years) and the median of B-hCG serum levels at diagnosis was 2319 IU. The vaginal bleeding and abdominal pain (27 [42.9\%]) were the most common complaints, followed by a delayed menstrual cycle (8 [12.7\%]). The majority of patients were subjected to surgical treatment and 29 cases $(46 \%)$ were treated by medical approaches. The median time to B-hCG resolution was 42 days. The Cox proportional hazards model showed the significant effect of the treatment approach on time to $\mathrm{B}-\mathrm{hCG}$ resolution $\left(\chi^{2}=37.78, \mathrm{df}=4, P<0.0001\right)$. Finally, the MTX plus surgery was the most effective treatment approach $(\mathrm{OR}=10.56, P<0.0001)$ in managing CSP patients.

Conclusions: Our findings in line with previous studies showed the superiority of the surgical approach alone or in combination with medical treatments compared to medical approaches alone.

Keywords: Cesarean scar pregnancy, Hysteroscopy, B-hCG resolution, Ectopic pregnancy
\end{abstract}

\section{Introduction}

Ectopic pregnancy in cesarean section scar (CSP) is the implantation of fertilized ovum within the myometrium at the site of the previous CS scar. Although it could be caused by uterine endometrial defect due to rough curettage, mycetoma metroplasty, hysteroscopy, or placental hand removal. There are 2 types of CSP including replacing the gestational sac (GS) in the scar and progressing to the uterus cavity and progressing the GS to the myometrium and uterine serosa (1). About $70 \%$ of CSP cases are women with a history of more than 2 CS while it can even be observed in people with a history of one CS. The overall incidence of CSP is one per 1800 to one per 2200 pregnancies, but its incidence is rising due to an increase in CS. In addition, the CSP is a potentially life-threatening condition and leads to uterus rupture, severe bleeding, disseminated intravascular coagulation, and maternal death if it is not diagnosed timely (2). Transvaginal ultrasonography has a sensitivity of $86 \%$ for CSP diagnosing in the first trimester. On the other hand, other diagnostic methods including Doppler ultrasound, 3D ultrasound, magnetic resonance imaging, hysteroscopy, and cystoscopy are used in cases where ultrasound is ambiguous (3).
Treatment is selected based on patient conditions, the viability of the pregnancy, the gestational age, and the desire for the next pregnancy. In general, the treatments used for the CSP include (4):

1. Methotrexate (MTX): Single or multiple doses or topical (the intravenous injection of gel);

2. Embryocidals: potassium chloride injection, vasopressin, glucose with osmotic fluid or the injection of crystalline trichosanthin into the GS;

3. Aspiration of the GS;

4. Combination therapy: MTX + Aspiration and other combinations of the above (e.g., administration of MTX with embryocidals);

5. Curettage;

6. Surgery using laparoscopy, laparotomy, hysteroscopy, and hysterectomy;

7. Uterine artery embolization (UAE) with gelatinous sponges.

Limited studies evaluated the efficacy and safety of different therapeutic approaches in managing CSP patients. Hence, there is still no consensus on its treatment long after the CSP explanation. Accordingly, the present study aimed to examine the effectiveness of various treatment modalities in patients with CSP based on the 
human chorionic gonadotropin (hCG) resolution time in order to find the most effective treatment in CSP patients.

\section{Materials and Methods}

Study Design

This cross-sectional study was carried on patients suspicious to CSP referring to Imam Khomeini Hospital, Ahvaz from Jun 2016 to March 2018. The inclusion criterion was confirmed diagnosis of CSP and patients with acute severe blood loss, hypovolemic shock, renal failure, active pelvic infection, and those with coagulation disorders were excluded from the study. Then, patients' demographic information was extracted from the files.

\section{Measurements}

Electrophysiology diagnosis was performed based on history, clinical examinations, and serum B-hCG level. The B-hCG serum level of more than $25 \mathrm{IU}$ is considered positive. Serum $\beta$-hCG levels were measured by the ELISA method and CSP diagnosis was confirmed using abdominal or vaginal ultrasonography by an expert radiologist. Further, hemodynamic factors and the B-hCG serum level were checked weekly, and the patients were followed-up until B-hCG resolution.

\section{Interventions}

Based on our internal protocol, patients were treated with different approaches. Medical approaches included systemic MTX administration and systemic MTX plus intra sac injection. Furthermore, surgical procedures were curettage, hysteroscopy, UAE, lower uterine segment and hysteroscopy, hysterectomy, curettage along with embolization and hysterectomy, and embolization. Some cases treated with medical and surgical combination therapy encompasses surgery plus MTX and MTX plus surgery plus intrasac injection. Eventually, four-dose of one $\mathrm{mg} / \mathrm{kg}$ of MTX was prescribed for patients.

\section{Outcomes}

The primary outcome in the study was time interval from treatment to hCG resolution and the secondary outcome was hospitalization time.

\section{Statistical Analysis}

All variables were subjected to descriptive analysis including mean, standard deviation, and frequency. The mean time to event was calculated by the Kaplan-Meier plot. Moreover, univariate and multivariable analyses of time to event data were performed using the Cox proportional hazards model. Additionally, the assessment proportional hazard assumption was performed using the score process plot and Kolmogorov-type supremum test $(P=0.05)$. The computations were performed using SPSS software, version 19 (Statistical Package for Social Service Inc., Chicago, IL, USA) and the significant level for univariate and multivariable analyses were 0.20 and 0.05 , respectively.

\section{Results}

In general, 63 patients with a confirmed diagnosis of CSP were recruited in this study. The mean age of the subjects was $34.2 \pm 5.1$ years (in the range of 22 to 44 years). Additionally, gravity, parity, and abortion means were $4,2.3$, and 0.6 , respectively. Similarly, the median of the CS interval was 16 months and the median of B-hCG serum levels at diagnosis was 2319 IU. The mean time of hospitalization was 9.8 days (Table 1) as well. In addition, the most common complaint was vaginal bleeding and abdominal pain (27 [42.9\%]), followed by a delayed menstrual cycle (8 [12.7\%]). The initial sonography showed electrophysiology (EP) line in $90.5 \%$ of the patients and fetal heart rate (FHR) was detected in 18 cases $(28.6 \%)$. The majority of patients were subjected to surgical treatment and 29 cases (46\%) were treated by medical approaches (Table 2). Further, hysteroscopy was the most common surgical approach for managing the CSP in our center. Three patients were treated by the lower uterine segment resection through laparotomy following hysteroscopy due to uncontrollable hemorrhage and severe placental adhesion. A patient who was under treatment by the multiple-dose of MTX, was discharged with personal consent, and subsequently readmitted

Table 1. Patient's Characteristics

\begin{tabular}{|c|c|c|c|c|c|c|c|c|}
\hline Variables & Minimum & 25\% Percentile & Median & 75\% Percentile & Maximum & Mean & SD & $\begin{array}{c}\text { Normality Test Passed } \\
(\text { alpha }=0.05)\end{array}$ \\
\hline Age & 22.00 & 30.00 & 34.00 & 39.00 & 44.00 & 34.22 & 5.135 & Yes \\
\hline Gravidity & 2.000 & 3.000 & 4.000 & 5.000 & 8.000 & 4.016 & 1.508 & No \\
\hline Parity & 1.000 & 2.000 & 2.000 & 3.000 & 7.000 & 2.333 & 1.191 & No \\
\hline Abortion & 0.0 & 0.0 & 0.0 & 1.000 & 5.000 & 0.6825 & 1.060 & No \\
\hline Curettage & 0.0 & 0.0 & 0.0 & 1.000 & 3.000 & 0.3333 & 0.6476 & No \\
\hline Cesarean section & 1.000 & 1.000 & 2.000 & 3.000 & 4.000 & 2.079 & 0.8289 & Yes \\
\hline Cesarean section interval & 6.000 & 16.00 & 29.00 & 39.00 & 120.0 & 31.65 & 23.95 & No \\
\hline Diagnosis age & 4.000 & 6.000 & 7.000 & 8.000 & 13.00 & 7.349 & 1.761 & No \\
\hline Initial B-hCG & 5.000 & 2319 & 6620 & 14667 & 77435 & 12236 & 15887 & No \\
\hline Time to B-hCG resolution & 1.000 & 28.00 & 42.00 & 56.00 & 82.00 & 41.60 & 16.96 & Yes \\
\hline Hospitalization time & 3.000 & 5.000 & 10.00 & 14.00 & 26.00 & 9.871 & 5.161 & Yes \\
\hline
\end{tabular}




\begin{tabular}{|c|c|c|c|c|}
\hline Variables & & No. & $\%$ & $\begin{array}{c}\text { Valid } \\
\text { Percent }\end{array}$ \\
\hline \multirow{4}{*}{$\begin{array}{l}\text { Clinical } \\
\text { presentations }\end{array}$} & Vaginal bleeding & 27 & 42.9 & 42.9 \\
\hline & Delayed menstrual cycles & 8 & 12.7 & 12.7 \\
\hline & $\begin{array}{l}\text { Vaginal bleeding and } \\
\text { abdominal pain }\end{array}$ & 1 & 1.6 & 1.6 \\
\hline & Abdominal pain & 27 & 42.9 & 42.9 \\
\hline \multirow{2}{*}{ FHR } & Yes & 18 & 28.6 & 28.6 \\
\hline & No & 45 & 71.4 & 71.4 \\
\hline \multirow{5}{*}{ Treatment } & MTX systemic & 21 & 33.3 & 33.3 \\
\hline & $\begin{array}{l}\text { MTX systemic and intra sac } \\
\text { injection }\end{array}$ & 8 & 12.7 & 12.7 \\
\hline & Surgery & 18 & 28.6 & 28.6 \\
\hline & Surgery + MTX & 13 & 20.6 & 20.6 \\
\hline & $\begin{array}{l}\text { MTX + Surgery + Intrasac } \\
\text { injection }\end{array}$ & 3 & 4.8 & 4.8 \\
\hline \multirow{8}{*}{$\begin{array}{l}\text { Surgical } \\
\text { intervention }\end{array}$} & Non-surgical treatments & 29 & 46.0 & 46.0 \\
\hline & Curettage & 4 & 6.3 & 6.3 \\
\hline & Hysteroscopy & 21 & 33.3 & 33.3 \\
\hline & UAE & 2 & 3.2 & 3.2 \\
\hline & $\begin{array}{l}\text { Lower uterine segment } \\
\text { resection and hysteroscopy }\end{array}$ & 3 & 4.8 & 4.8 \\
\hline & Hysterectomy & 2 & 3.2 & 3.2 \\
\hline & $\begin{array}{l}\text { Curettage and embolization } \\
\text { and hysterectomy }\end{array}$ & 1 & 1.6 & 1.6 \\
\hline & Embolization & 1 & 1.6 & 1.6 \\
\hline \multirow{3}{*}{$\begin{array}{l}\text { Initial } \\
\text { sonography }\end{array}$} & Cesarean scar pregnancy & 57 & 90.5 & 90.5 \\
\hline & Missed abortion & 5 & 7.9 & 7.9 \\
\hline & $\begin{array}{l}\text { Retained products of } \\
\text { conception }\end{array}$ & 1 & 1.6 & 1.6 \\
\hline
\end{tabular}

Note. FHR: fetal heart rate; MTX: Methotrexate.

with severe hemorrhage and subjected to hysterectomy. Furthermore, a patient with a history of EP line according to ultrasound examination was admitted due to severe hemorrhage and immediately subjected to diagnostic suction curettage, but hysterectomy was carried out because of the inability to control the hemorrhage.

The median time to B-hCG resolution was 42 days (Table 3, Figure 1A). The Cox proportional hazards model showed the significant effect of treatment approach on time to B-hCG resolution $\left(\chi^{2}=37.78, \mathrm{df}=4, P<0.0001\right)$. Additionally, the MTX plus surgery was the most effective treatment approach $(\mathrm{OR}=10.56, P<0.0001)$ in managing CSP patients (Table 3, Figure $1 \mathrm{~B})$. The curettage $(\mathrm{OR}=7.8$, $P<0.0001)$ and lower uterine segment and hysteroscopy
Table 4. Hospitalization Time in Different Treatment Approaches

\begin{tabular}{lccc}
\hline Treatment & Estimate & \multicolumn{2}{c}{ Cox Regression Model } \\
\cline { 3 - 4 } Approaches & Time (day) & OR & P value \\
\hline Medical* & 12.310 & & \\
Medical + surgery & 11.6 & $0.94(0.47-1.8)$ & 0.87 \\
Surgery & 4.5 & $17(6.2-48)$ & $<0.0001$ \\
Overall & 9.871 & & \\
\hline
\end{tabular}

Note. *Baseline; OR: odds ratio.

$(\mathrm{OR}=0.01, P<0.0001)$ significantly improved the B-hCG resolution time compared with non-surgical treatments (Figure 1C).

The median hospitalization time was 9.87 days. Moreover, the Cox proportional hazards model demonstrated the significant effect of the treatment approach on hospitalization time $\left(\chi^{2}=53.83, \mathrm{df}=2\right.$, $P<0.0001)$. The least hospitalization time was found in patients undergoing surgery $(\mathrm{OR}=17, P<0.0001)$, the related data are shown in Table 4 and Figure 1D.

Based on the Spearman test, a significant correlation was observed between initial sonography, FHR, intra sac injection, and initial B-hCG with time to B-hCG resolution. The factors were analyzed with univariate and multivariate regression models. Accordingly, the initial sonography and initial B-hCG were determined as independent predictors of time to B-hCG resolution (Table 5).

\section{Discussion}

Larsen et al introduced the first case of CSP (5). Various theories were raised about the CSP etiology. One of these theories is the implantation and migration of the embryo through a microscopic wedge or fistula defect in the uterus on the site of the cesarean section (CS) and the fetal infiltration to the myometric site of the scar (6). Another theory, proposed by Ben-Nagi et al, is endometrial changes in the area of CS that reduces vascularization and low cesarean site leukocytes compared to non-cesarean groups (7). Some studies described the expansion of GS on the site of CS by endogenous (the growth of the sac toward the endometrium and uterine cavity) or exogenous (the expansion and penetration of the GS toward the myometrium and serous) methods (8). CSP treatment methods are very diverse and rely on expectant and surgical procedures.

The common symptoms of CSP include vaginal bleeding with or without pain, hemorrhagic shock, and uterine rupture (9). Other symptoms are lower abdominal

Table 3. Time to B-hCG Resolution

\begin{tabular}{|c|c|c|c|c|c|c|c|}
\hline \multirow{2}{*}{ Mean } & \multirow{2}{*}{ Std. Error } & \multicolumn{2}{|c|}{$95 \% \mathrm{Cl}$} & \multirow{2}{*}{ Median } & \multirow{2}{*}{ Std. Error } & \multicolumn{2}{|c|}{$95 \% \mathrm{Cl}$} \\
\hline & & Lower Bound & Upper Bound & & & Lower Bound & Upper Bound \\
\hline 41.603 & 2.137 & 37.415 & 45.791 & 42.000 & 4.521 & 33.138 & 50.862 \\
\hline
\end{tabular}

Note. B-hCG: Beta-human chorionic gonadotropin. 

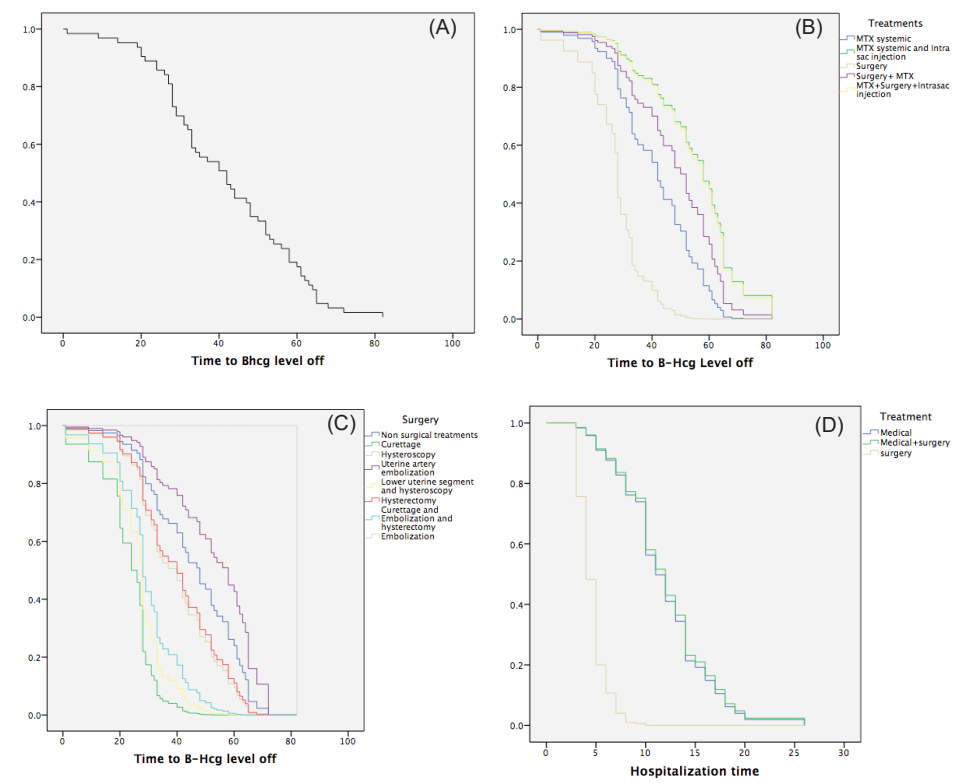

Figure 1. (A) Time to B-hCG Resolution. (B) Comparison of Treatment Approach Efficacy. (C) Comparison the Effect of Surgical Procedures With Non-surgical Treatments on B-hCG Resolution Time. (D) Comparison the Effect of Surgical Procedures With Non-surgical Treatments on Hospitalization Time

pain and diarrhea (10). In a study on 57 patients, $37 \%$, $38 \%, 16 \%$, and $9 \%$ of cases were related to asymptomatic, painless bleeding, bleeding with pain, and abdominal pain without bleeding, respectively (11). Similarly, the most common clinical symptoms in our studio patients were vaginal bleeding and abdominal pain.

Our findings indicated that the MTX plus surgery could be considered as the most effective treatment approach in managing CSP patients, with the shortest hospitalization time in patients undergoing surgical treatment. Hysteroscopy was the most common surgery in our patients. So far, hysteroscopy has been suggested as the most widely accepted procedure for the treatment of several gynecological disorders (12-14). Moreover, several investigators in recent years have presented the successful management of CSPs by hysteroscopy alone or in combination with other surgical or medical methods (15-17). During the present study, no complications were reported after the hysteroscopic intervention, indicating that this method was effective and safe in managing CSP patients. Compared to non-surgical treatments, curettage was the most effective approach in terms of the time to B-hCG resolution that was due to the low initial B-hCG in this group of patients. Contrarily, Petersen et al reviewed the treatment approach in managing CSP and found that the interventional approach was most effective compared to the medical approach. They further reported the use of the laparoscopic surgical approach as the most effective treatment for CSP patients (9). It should be mentioned that no laparoscopic surgery was performed on our studied patients because all of them were at high risk. Similarly, Mahout et al. in a systematic review study analyzed 3225 cases and reported a $62 \%$ success rate in systematic MTX and/or the local injection of MTX or potassium chloride. However, they found $12 \%$ unsuccessful treatment by the hysteroscopic resection of CSP and expectant management led to a $57 \%$ live birth rate, but $63 \%$ of women needed hysterectomy (18). The 2 last systematic review studies showed the insufficiency of medical treatment alone in managing the CSP $(9,18)$. A special treatment proposal for these patients is impossible given that the choice of therapeutic approach in patients with CSP completely closed the ultrasound view, the patient's condition and desire. Hence, judgment about the best treatment in managing the CSP patients is difficult due to the limitation in setting up a randomized clinical trial study. However, based on the findings of our study, surgical or surgical in combination with medical approaches not only reduced

Table 5. Univariate and Multivariate Regression Model

\begin{tabular}{lccc}
\hline \multirow{2}{*}{ Variables } & \multicolumn{2}{c}{ Univariate } & \multicolumn{1}{c}{ Multivariable } \\
\cline { 2 - 4 } & OR (95\% Cl) & $\boldsymbol{P}$ Value & Adjusted OR (95\% Cl) \\
\hline FHR & $-15.95(-24.5,-7.33)$ & $<0.0001$ & $-3.96(-14,6.07)$ \\
Intra sac injection & $8.9(4.35,13.54)$ & $<0.0001$ & $4.51(-0.76,9.79)$ \\
Initial B-HCG & $0.001(0,0.001)$ & $<0.0001$ & $0.001(0,0.001)$ \\
Initial sonography & $-15.59(-26.8,-4.3)$ & $<0.0001$ & $-10.71(-20.4,-0.97)$ \\
\hline
\end{tabular}

Note. FHR: Fetal heart rate; B-hCG: Beta-human chorionic gonadotropin; OR: Odds ratio; $\mathrm{Cl}$ : Confidence interval. 
the duration of hospitalization but also provided the shortest B-hCG resolution time.

Based on our findings, the initial sonography in more than $90 \%$ of patients showed the EP-line. Moreover, our multivariate regression model indicated the initial sonography as an indecent predictive factor for B-hCG resolution time. Additionally, the most important factor in choosing a therapeutic approach in CSPs is the initial ultrasonic pattern. Therefore, due to the importance of ultrasonography in the diagnosis and treatment of CSP, the GS ultrasound evaluation at the 6th week of pregnancy is suggested in patients with previous CS in order to prevent the late complications of non-diagnosis of the CSP.

In general, our findings in line with those of previous studies showed the superiority of surgical approach, alone or in combination with medical treatments, rather than medical approaches alone. The need for transfusion, mortality, and subsequent fertility were not evaluated during the study and this was the main limitation of our study. Accordingly, the evaluation of the other CSP complications such as residual mass size, transfusion rate, and subsequent fertility is suggested for future studies

\section{Conflict of Interests}

Authors declare that they have no conflict of interests.

\section{Ethical Issues}

This study was approved by the Ethics Committee of Ahvaz Jundishapur University of Medical Sciences (IR.AJUMS. REC.1396.1138). Prior to entering the study, patients were provided with necessary explanations and were requested to sign the informed consent. Written informed consent was obtained from all patients and normal individuals.

\section{Financial Support}

We had no special financial support.

\section{Acknowledgments}

We wish to thank all our colleagues at Razi Hospital, Ahvaz, Iran.

\section{References}

1. Patel MA. Scar ectopic pregnancy. J Obstet Gynaecol India. 2015;65(6):372-375. doi:10.1007/s13224-015-0817-3

2. Grechukhina O, Deshmukh U, Fan L, et al. Cesarean Scar Pregnancy, Incidence, and Recurrence: Five-Year Experience at a Single Tertiary Care Referral Center. Obstet Gynecol. 2018;132(5):1285-1295. doi:10.1097/ aog.0000000000002940

3. Osborn DA, Williams TR, Craig BM. Cesarean scar pregnancy: sonographic and magnetic resonance imaging findings, complications, and treatment. J Ultrasound Med. 2012;31(9):1449-1456. doi:10.7863/jum.2012.31.9.1449

4. Litwicka K, Greco E. Caesarean scar pregnancy: a review of management options. Curr Opin Obstet Gynecol. 2011;23(6):415-421. doi:10.1097/GCO.0b013e32834cef0c

5. Larsen JV, Solomon MH. Pregnancy in a uterine scar sacculus--an unusual cause of postabortal haemorrhage. A case report. S Afr Med J. 1978;53(4):142-143.

6. Marchiolé P, Gorlero F, de Caro G, Podestà M, Valenzano $\mathrm{M}$. Intramural pregnancy embedded in a previous Cesarean section scar treated conservatively. Ultrasound Obstet Gynecol. 2004;23(3):307-309. doi:10.1002/uog.981

7. Ben-Nagi J, Walker A, Jurkovic D, Yazbek J, Aplin JD. Effect of cesarean delivery on the endometrium. Int J Gynaecol Obstet. 2009;106(1):30-34. doi:10.1016/j.ijgo.2009.02.019

8. Vial Y, Petignat P, Hohlfeld P. Pregnancy in a cesarean scar. Ultrasound Obstet Gynecol. 2000;16(6):592-593. doi:10.1046/j.1469-0705.2000.00300-2.x

9. Birch Petersen K, Hoffmann E, Rifbjerg Larsen C, Svarre Nielsen H. Cesarean scar pregnancy: a systematic review of treatment studies. Fertil Steril. 2016;105(4):958-967. doi:10.1016/j.fertnstert.2015.12.130

10. Obed S. Diagnosis of unruptured ectopic pregnancy is still uncommon in Ghana. Ghana Med J. 2006;40(1):3-7.

11. Silver RM, Landon MB, Rouse DJ, et al. Maternal morbidity associated with multiple repeat cesarean deliveries. Obstet Gynecol. 2006;107(6):1226-1232. doi:10.1097/01. aog.0000219750.79480.84

12. Camanni M, Bonino L, Delpiano EM, Ferrero B, Migliaretti G, Deltetto F. Hysteroscopic management of large symptomatic submucous uterine myomas. J Minim Invasive Gynecol. 2010;17(1):59-65. doi:10.1016/j.jmig.2009.10.013

13. Guin G, Sandhu SK, Lele A, Khare S. Hysteroscopy in evaluation of abnormal uterine bleeding. J Obstet Gynaecol India. 2011;61(5):546-549. doi:10.1007/s13224-011-0088-6

14. Daniilidis A, Pantelis A, Dinas $K$, et al. Indications of diagnostic hysteroscopy, a brief review of the literature. Gynecol Surg. 2012;9(1):23-28. doi:10.1007/s10397-0110695-3

15. Deans R, Abbott J. Hysteroscopic management of cesarean scar ectopic pregnancy. Fertil Steril. 2010;93(6):1735-1740. doi:10.1016/j.fertnstert.2008.12.099

16. Wang CJ, Tsai F, Chen C, Chao A. Hysteroscopic management of heterotopic cesarean scar pregnancy. Fertil Steril. 2010;94(4):1529.e1515-1528. doi:10.1016/j. fertnstert.2010.02.039

17. Qian ZD, Huang LL, Zhu XM. Curettage or operative hysteroscopy in the treatment of cesarean scar pregnancy. Arch Gynecol Obstet. 2015;292(5):1055-1061. doi:10.1007/ s00404-015-3730-1

18. Maheux-Lacroix S, Li F, Bujold E, Nesbitt-Hawes E, Deans R, Abbott J. Cesarean scar pregnancies: a systematic review of treatment options. J Minim Invasive Gynecol. 2017;24(6):915-925. doi:10.1016/j.jmig.2017.05.019

(c) 2020 The Author (s); This is an open-access article distributed under the terms of the Creative Commons Attribution License (http://creativecommons.org/licenses/by/4.0), which permits unrestricted use, distribution, and reproduction in any medium, provided the original work is properly cited. 